\title{
Monitoring of Drainage System in Urban Using Device Free Localization Neural Networks and Cloud computing
}

\author{
Mr. Rahul Sharma \\ Auricle Technologies Bikaner Rajasthan India \\ rahulmnu1@gmail.com
}

\begin{tabular}{|c|c|}
\hline Article History & Abstract \\
\hline $\begin{array}{l}\text { Article Submission } \\
29 \text { August } 2018 \\
\text { Revised Submission } \\
22 \text { October } 2018 \\
\text { Article Accepted } \\
30 \text { November } 2018 \\
\text { Article Published } \\
31^{\text {st }} \text { December } 2018\end{array}$ & $\begin{array}{l}\text { Wireless Sensor Network is a Wi-Fi community consisting of spatially propagated } \\
\text { and self-sufficient devices using sensors to detect physical or environmental } \\
\text { conditions. During heavy rainfall, the urban drainage system cannot drain the water. } \\
\text { A wireless sensor with many interconnected wireless sensor nodes captures real-time } \\
\text { data from the network environment and transmits this data to a base station for } \\
\text { analysis and operation. With wireless sensor nodes, it is possible to capture and } \\
\text { monitor the amount of water in drainages and the difference in water flow between } \\
\text { the two points in the drainage system. Nevertheless, the majority localization } \\
\text { techniques aims on device based localization, which can find target with festinated } \\
\text { devices. It is not suitable for applications such as terrain, drainage flow and } \\
\text { flooding. Here device free wireless localization system using artificial neural } \\
\text { networks and a cluster based wireless sensor network system to monitor urban } \\
\text { drainage is proposed. There are two stages in the system. During the off-line } \\
\text { preparation stage, Acceptable Signal Strength (RSS) differential metrics are } \\
\text { calculated between the RSS metrics together while the monitor area is empty and } \\
\text { calculated by a specialized in the region. Some RSS dissimilarity values are selected } \\
\text { in the RSS Difference Matrix. The RSS dissimilarity standards and associated matrix } \\
\text { indices are taken as the inputs of the ANN representation in addition to the identified } \\
\text { position coordinate are in its outputs. The real-time data collected from the wireless } \\
\text { sensor network is used to detect overflow and provide alarms before disturbances } \\
\text { arise. } \\
\text { Keywords: Urban, Network, Protocol, Sensor, Localization }\end{array}$ \\
\hline
\end{tabular}

\section{Introduction}

Remote sensors are equipped for estimating, registering, and imparting ecological information to different frameworks, where they can be comprehended as important data. A remote sensor arrange comprises of a lot of sensor hubs [1]. They gather the information and send it to the base station, where they break down, procedure and store the gathered information. Front line systems are bi-directional and permit sensor execution to be controlled. The improvement of remote sensor systems requires military projects, including battle observing; Today, such systems are utilized in numerous business and shopper capacities, including business style checking and control, device wellbeing following, and now remote sensors have locally available detecting, preparing, correspondence and capacity abilities to assist them with putting away and break down information. Incorporate and total own information gathered[2]. In the course of recent years, the urban waste framework has helped humanity to construct progressively present day urban communities. Much advancement has been made in deciding area and foreseeing precipitation in various districts. By and large sewage is stopped up and the conduit is hindered by a waste hindrance. Consequently, it is important to continually screen and keep up seepage frameworks to forestall flood. 
In the WSN, normally a few sensor hubs are mounted in the checking territory. These sensor hubs interface through remote correspondence and complete detecting, identification and checking undertakings in a community oriented way. With abilities of detecting, registering and correspondence, WSN is broadly utilized in numerous regions, for instance, indoor fire discovery, object following, reconnaissance detecting and building security checking. In these applications, restriction plays a significant and significant job in WSNs. The satellite-based confinement framework gives a good area based help (LBS) to clients in the dour condition. The cell based framework can ascertain the areas of cell phone clients, while the cell based framework as a rule experiences enormous restriction mistakes

Most limitation frameworks that utilization remote neighborhood (WLANs) can give LBS to clients while choosing WLAN terminal gadgets. Bright Band (WWB) Localization System Users must take WWB labels to ascertain their positions. Advanced mobile phone sensors are utilized for route and restriction administrations. Despite the fact that the infrared-based limitation framework doesn't require terminal gear; nature around execution is effortlessly influenced. Be that as it may, one of the difficulties of gadget free remote confinement in WSNs is the flightiness of RSS estimations in multipath conditions, particularly in complex situations where individuals are commonly moving[3]. To address this issue, in this paper, we allude to the well known WLN unique mark restriction technique and propose a gadget free remote limitation framework utilizing fake neural systems (ANN).

\section{Literature Survey}

Remote sensor systems are generally used to catch information from our outside surroundings. This information empower us to screen and evaluate ecological varieties and to avoid potential risk when a looming flood, tidal wave and seismic tremor happens. The little size of remote sensors empowers checking of varieties in pressure, light, vibration, tilt, and clamor in zones that are for all intents and purposes unavailable. There are a few water stream models for ascertaining the most extreme release [4]. By deciding the most elevated level of water in a territory, the size and plan prerequisites of the seepage can be coordinated. The accompanying area fundamentally talks about the judicious technique and two well known water stream models utilized in the city, the Saint-Vennant strategy. The sane technique is utilized to figure the aggregate sum of water stream amassing in a stream. The computation of normal precipitation power utilized in a discerning way depends on the examination of chronicled information of precipitation force over the previous year. Seepage checking arrangement of the Fisheries in the unified realm. The fundamental goal is to evaluate the annexed flood. These situations are not feasible because of wasteful estimation, which brings about off base outcomes progressively [5]. The arrangement is to gather a lot of continuous information and align the hydrodynamic model for the Berg Farm.

The stage utilized EJB innovation to make an undertaking level framework that gives free, arrange bolstered security, runtime conditions, and remote exchanges. The framework utilizes three layered engineering to serve numerous capacities [6]. These incorporate the dynamic organization of the pressure driven model, urban seepage arrange streamlining structure and arranging, visual data the executives, dynamic state examination and constant checking of the urban waste framework. QoS is frequently characterized as the target proportion of system benefits regarding data transfer capacity, deferral, unwavering quality and shrinkage. As such, the QoS objective is to convey high need parcels to a goal with high dependability and low dormancy [7]. Backing for QoS for WSNs has likewise been investigated in the writing and has recommended a few QoS-mindful MAC and steering conventions. Nonetheless, they just spotlight on certain QoS parameters, for example, postponement and reliability. Some natural uses of sensor systems incorporate checking the development of flying creatures, little creatures and bugs, and observing ecological conditions influencing plants and animals [8]Irrigation full scale contraptions for huge scope land following and planetary investigation of farming, organic, topographical and ecological contamination in the ocean, soil and condition.

The RTI-based limitation strategy delineates RSS lessening brought about by modest and basic equipment targets. They propose a regularization technique for clamor decrease and a measurable model that relates with 
the spatial areas of movement for dynamic picture estimation [9]. Because of the near exhibition of this technique, confinement without an information driven gadget has been widely explored. RTI-based gadget free limitation utilizing the division calculation and the associated segment naming calculation for target follows. Here certain hubs are set on the roof and the following region is isolated into various sub frames. For every subroutine, they utilized the Support Vector Regression (SVR) model to figure confinement organizes. Analysts have proposed a remote confinement framework without a gadget dependent on a radio guide. They determined the confinement results utilizing the probability strategy. They proposed another tool less restriction technique utilizing molecule filtration. With probabilistic characterization techniques dependent on non-biased investigation, it has molded the issue of instrumental restriction and limited the mistakes brought about by the multipath impact [10]. Unique mark limitation strategy works best in multipath situations. It is concluded that drainage is monitored with wireless sensor network but with the help of localized devices.

\section{Proposed Methodology}

In our proposed strategy, the neighborhood restriction framework sensor hubs are adjusted along the edges of the rectangular observing region. At the point when you go to the objective observing region, some remote connections between the sensor hubs conceal. At the point when you move to another area in the objective observing zone, there will be related remote connections and the shade. This is the reason a urban waste observing framework is required, which incorporates a few sensor hubs. Sensor hubs are put in touchy regions with the goal that the itemized status of urban seepage can be observed. Sensor hubs send information replicated to the base station. From that point, the information is sent to a server that is incorporated and associated with the checking framework. The computational consequences of the gathered information are sent to the front of the presentation. Water level sensors are utilized to gauge water levels in a seepage framework. Water level sensors can quantify the separation between the base surface of the channel and the water surface. Water stream meters are utilized to quantify water stream in a channel. All sensor hubs in the WSN measure RSS information and send information to the confinement server by means of the sink hub as shown in figure 1. An expert stands at select areas with notable area directions and some remote connections are concealed while being proficient at every area. We can get RSS grids similarly and figure RSS contrast measurements. We build up a straight capacity connection between the directions of the RSS contrast esteem data and the position ANN model.

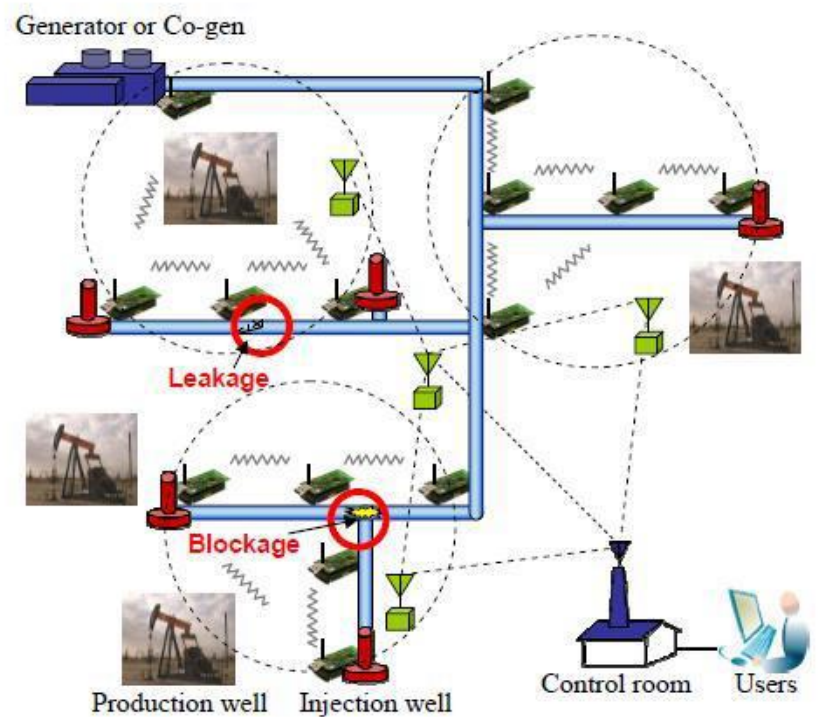

Fig 1: Drainage Pumping and flow path

This ANN representation is worn to estimate the position coordinates of the objective in the monitoring area. More specifically, the projected device liberated wireless localization system has 2 stages: off-line teaching and on-line localization. During the off-line preparation phase, RSS data is collected from the space monitoring area 
for some time. The data of all the sensor nodes is sent to the main server via the routed node. The server captures Data and compiles the data into RSS dimensions. It selects several specific locations and records their location coordinates. A professional stands at each location, collects RSS data from all sensor nodes, and processes them the same way to obtain RSS measurements. RSS difference measurement is measured by RSS measurements collected by area experts when the monitoring area is empty. By combining these RSS difference values and their matrix indices, some RSS difference values are selected and integrated into the ANN model. On the other hand, the known position coordinates of the selected positions are taken as p p outputs of the ANN model. Therefore, the ANN model can be trained using this data and linear function to calculate the localization coordinates. During the on-line localization phase, some wireless links are shaded as they move into the target monitoring area. The localization server collects RSS data from all sensor nodes and compiles this RSS data into the RSS matrix. It also calculates the RSS difference matrix. Since the selected RSS difference values and their indices in the matrix are input to the trained ANN model, the target coordinates of the target can be calculated for the ANN model as shown in figure 2.

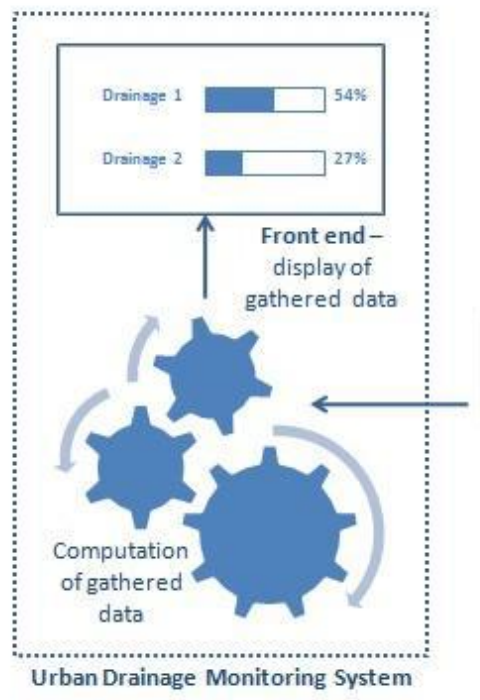

Urban Drainage Monitoring System

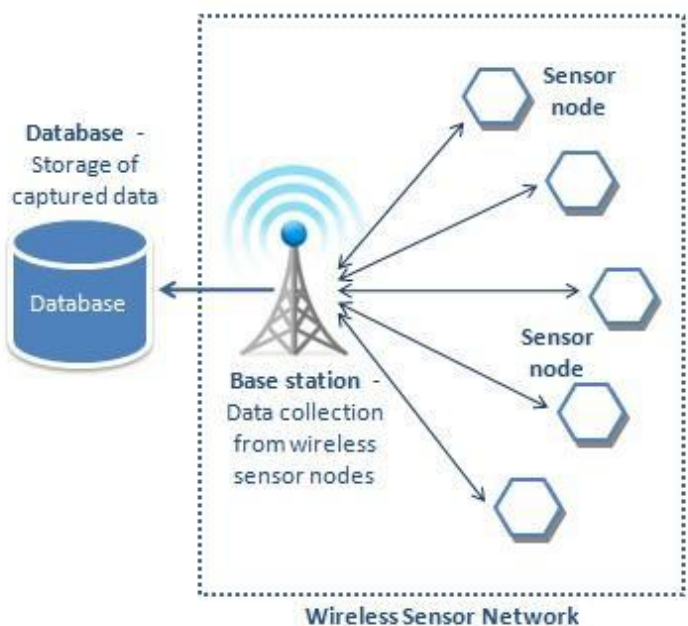

Wireless Sensor Network

Fig 2: Drainage interfaced with sensor network

In cases where impurities in the wastewater affect the sensor, the latter is placed on the metal crate. Therefore, waste in the drains is less likely to block the sensor or affect its readings. The proposed drainage monitoring system uses Arduino boards to monitor drainage by capturing the information of the sensor. Arduino is an easyto-use open source platform for hardware and software. It is a single-board microcontroller with a set of digital and analog input / output pins. The board uses the serial communication interface to easily transfer information between the board and any external devices such as personal computers or laptops. In our proposed framework, the RSS contrast esteems and related lattice records are incorporated into the contributions of the ANN model, whose $\mathrm{p}$ yields are position arranges. Via preparing the ANN model, we can compute the straight capacity between the sources of info and the p yields. At the point when we have to discover an objective in the checking zone, we utilize a prepared ANN model to compute the restriction directions of the objective.

\section{Simulation Results}

The simulations results are obtained by Contaiki Network Simulator. Here, if one of the wireless sensor transmits a signal then all the other wireless nodes will receive the same radio signals. In our method, the network consists of 24 sensor nodes, 2 sink node, and 2 localization server as shown in figure 3 . The 24 sensor nodes and calculate these RSS data. Purpose of sink node is to collect all data from remaining sensor nodes and at the same time it will transmit the same data to the device free localization network server. 


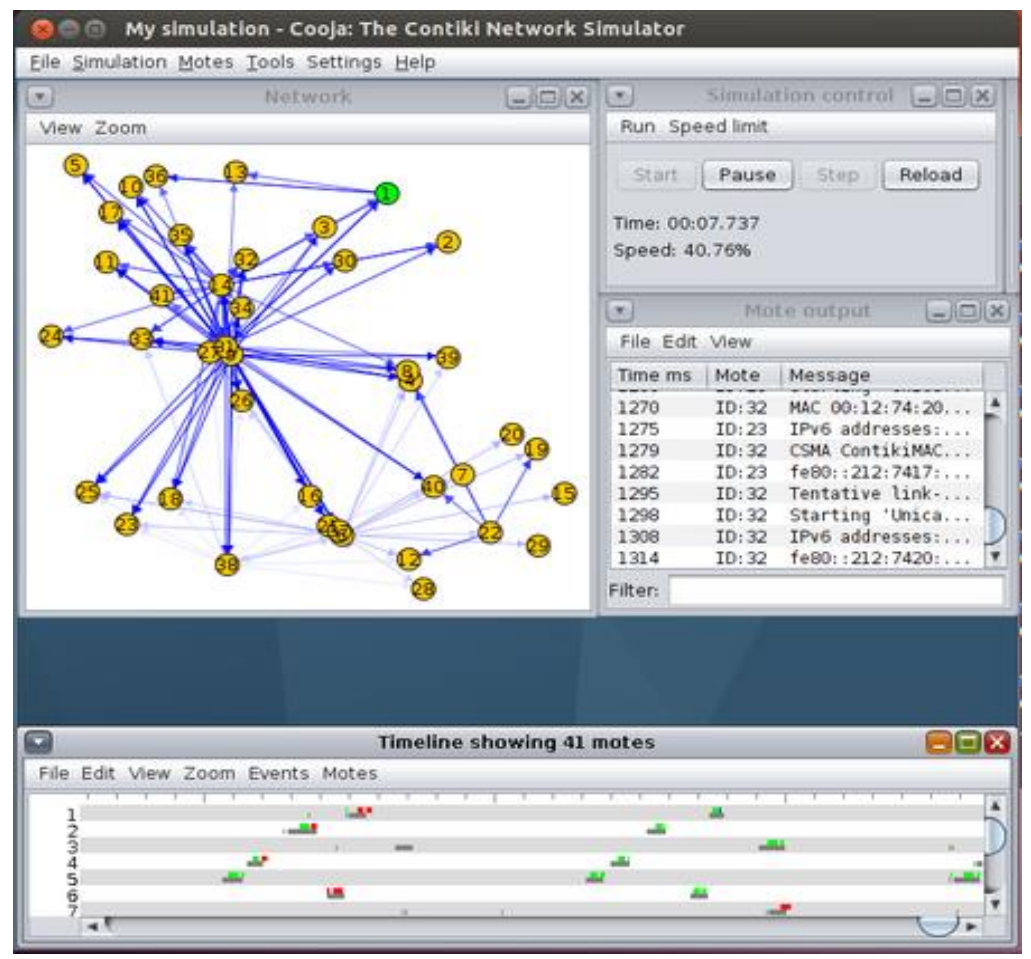

Fig 3: Sensor nodes set up

In the drainage monitoring area all the 24 nodes are evenly connected at the edges as demonstrated in figure 4 . Once the network is set up then the single node will broadcast such that remaining 23 nodes will receive and calculate the obtained RSS actual data shown in figure 5.

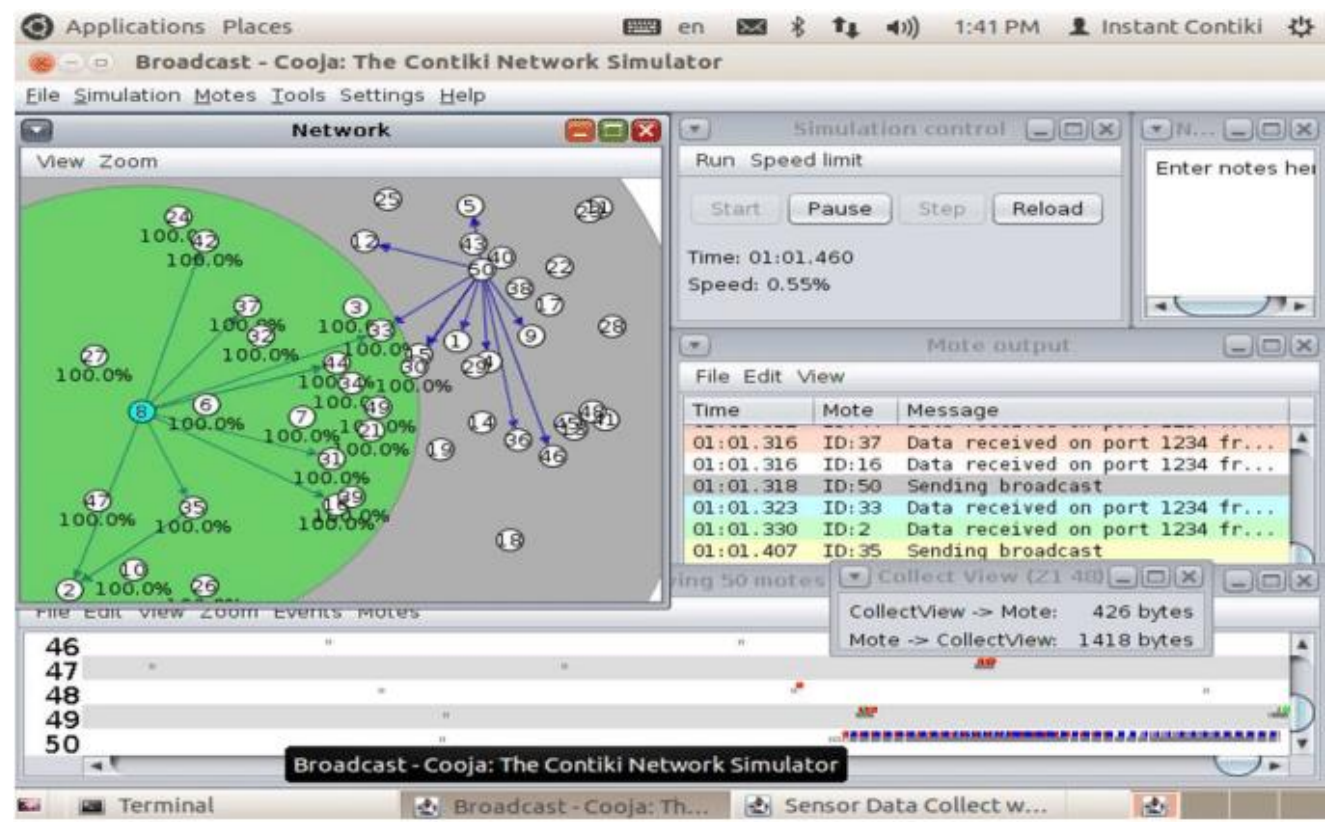

Fig 4: Sensor nodes of monitoring and duplication

The drainage runoff $7.40 \mathrm{~m} 3 / \mathrm{s}$ and $5.24 \mathrm{~m} 3 / \mathrm{s}$, which is considered to be larger than actual capacity of $5.72 \mathrm{~m} 3$ and $4.18 \mathrm{~m} 3$ in the region 3 and 6 respectively. It is concluded that regions 3 and 6 are exposed and level to brim over as shown in Table 1. 
TABLE I Drain-off range and actual capacity

\begin{tabular}{|c|c|c|}
\hline Monitoring Region & Drainage drimoff $\left(\mathbf{m}^{\mathbf{3}} / \mathbf{s}\right)$ & Actual Capacity $\left(\mathbf{m}^{\mathbf{3}}\right)$ \\
\hline 1 & 2.44 & 2.21 \\
\hline 2 & 4.70 & 2.17 \\
\hline 3 & 7.40 & 5.72 \\
\hline 4 & 5.24 & 2.24 \\
\hline 5 & 8.12 & 10.23 \\
\hline 6 & 5.24 & 4.18 \\
\hline 7 & 12.35 & 13.24 \\
\hline 8 & 11.01 & 12.32 \\
\hline
\end{tabular}

The simulation related to data in table 1 is shown in figure 5.

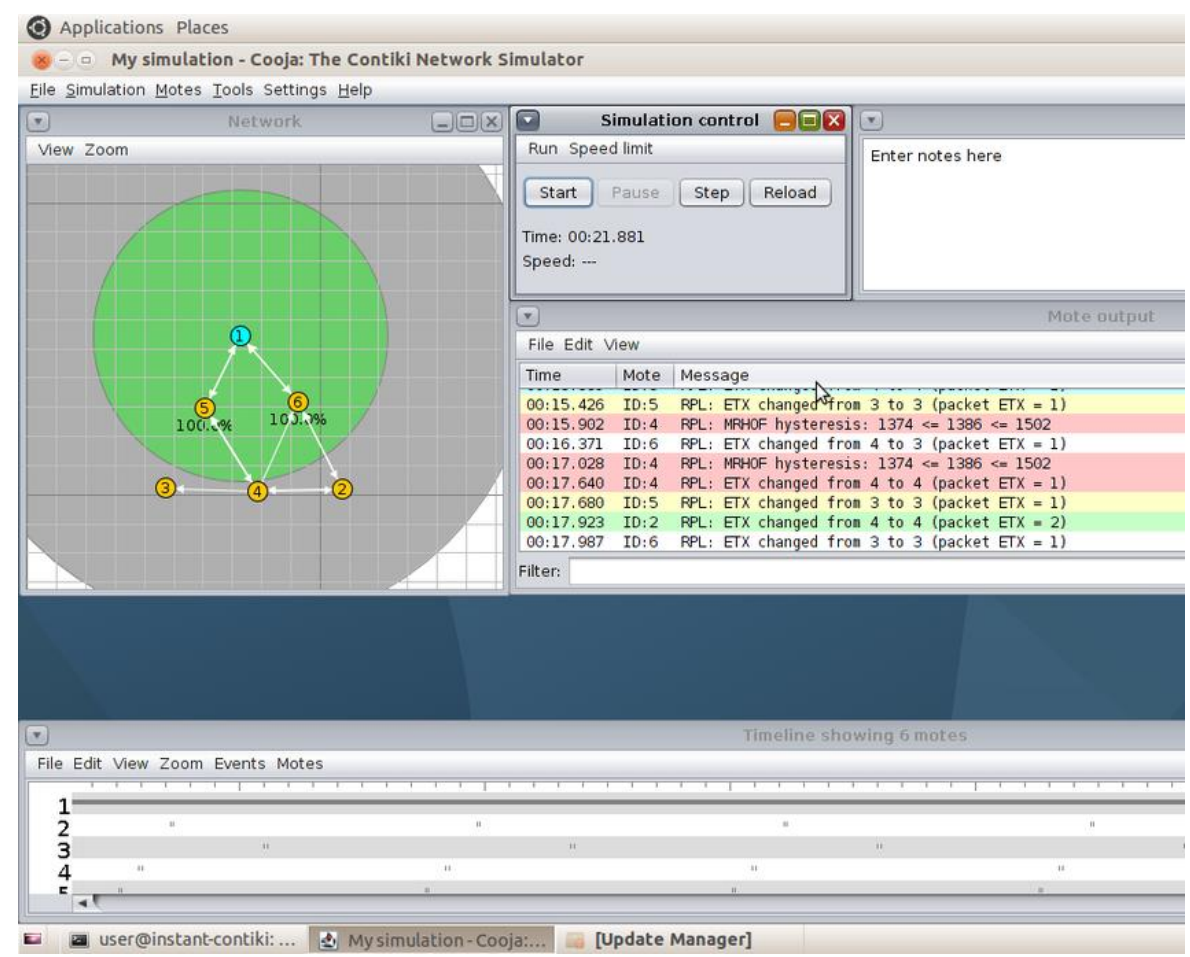

Fig 5: Broadcasting of Sensor nodes

\section{Conclusion}

Our proposed system is proved to be best among all the existing method from the above simulated results. Device free localization wireless sensors attracts powerful, dependable and reasonable and more over it gives the best option to observe atmosphere to keep away from disastrous situations. Henceforth the proposed framework is intended to identify ongoing instances of flood and deterrent in urban seepage by setting sensor hubs at vital areas inside the channels to screen water level and water-stream. The proposed checking 
framework permits early recognition of hindrance in the channels and nearby specialists can send the concerned group to proceed to clear the water-stream section before flood happens.

\section{References}

[1] J.Thomas, Wireless Sensor Networks, Netherlands: A John Wiley and Sons, Ltd, Second Edition, 2013.

[2] M. Erol-Kantarci and H.T. Mouftah, "Wireless sensor networks for cost-efficient residential energy management in the smart grid," IEEE Transactions on Smart Grid, vol. 2, no. 2, pp. 314-325, Jun,2011

[3] I. Al-Anbagi, M. Erol-Kantarci and H. T. Mouftah, "Delay Critical Smart Grid Applications and Adaptive QoS Provisioning,” IEEE Access, vol. 3, no. , pp. 1367-1378, 2016.

[4] Ralph, "Wireless Sensor Networks:" 2017 International Conference on Advanced Information Networking and Applications Workshops, Bankok, 2017, pp. 12-21.

[5] S. R. Jino Ramson and D. J. Moni, "Applications of wireless sensor networks - A survey," 2017 International Conference on Innovations in Electrical, Electronics, Instrumentation and Media Technology (ICEEIMT), Coimbatore, 2017, pp. 325-329.

[6] K. Lee and E. Song, "Wireless Sensor Network Based on IEEE 1451.0 and IEEE 1451.5-802.11," 2007 8th International Conference on Electronic Measurement and Instruments, Xi'an, 2007, pp. 4-7-51

[7] Shankar Jain and Hitesh Sharma "A Frame Work in Wireless Sensor Network," 2017 International Conference in Computing, Communication Control and Networking, India, 2017, pp. 23-29.

[8] Chen, J., Yatskiv, V., Sachenko, A. et al. Wireless sensor networks based on modular arithmetic. Radioelectron.Commun.Syst. 60, 215-224 (2017)

[9] Dos Santos, J., Hennebert C. and Lauradoux, C., 2015. Preserving privacy in secured ZigBee wireless sensor networks. Proceedings of the 2015 IEEE 2nd World Forum on Internet of Things (WF-IoT), Milan, pp. 715-720

[10] Kalis., and Rathilk, 2017. Malware contemporary composite Networks. Theory and Applications, Morgan Kaufmann, Cambridge, MA 